\title{
PRESENTACIÓN
}

\section{Cáncer de mama: un reto para la sociedad y los sistemas de salud}

$E_{l a d}^{1}$ cáncer de mama constituye un problema de salud pública y es la primera causa de incidencia y mortalidad por cáncer en la mujer adulta en Latinoamérica y en el ámbito mundial. El cáncer mamario dejó de estar circunscrito a los países desarrollados y a mujeres con mayores recursos económicos, incluso ha desplazado al cáncer cervicouterino en varias regiones de Latinoamérica como primera causa de muerte por neoplasias malignas femeninas. Nos encontramos ante un problema de gran magnitud, poco reconocido como tal en la región, cuyo impacto rebasa la salud de la mujer en lo individual, y afecta a la pareja, a la familia, a la sociedad y al sistema de salud, como señala Julio Frenk en el editorial que introduce este volumen.

Ante este panorama, nos propusimos coadyuvar a mejorar los programas de prevención y control del cáncer de mama a través de la evidencia científica. Nos complace informar la excelente respuesta de un grupo multidisciplinario que representa a varias instituciones y países, que incluye científicos prestigiados expertos en el tema, representantes de las organizaciones civiles, autoridades de salud, mujeres supervivientes, entre otros muchos actores. Todos ellos contribuyeron con su experiencia y quehacer profesional al brindar información actualizada que se presenta en este suplemento especial sobre cáncer mamario.

Las contribuciones que lo conforman se ubican en tres áreas principales: la perspectiva epidemiológica, que presenta, desde la óptica poblacional, el panorama de la enfermedad en términos de su magnitud y carga, sus tendencias a través del tiempo, y los factores de riesgo asociados a ella; el abordaje de los sistemas de salud, donde se discuten las barreras desde la demanda y la oferta, incluyendo la falta de infraestructura y recursos que condiciona un rezago en la detección oportuna, así como el tratamiento; y la participación de la sociedad civil a través de grupos organizados a nivel nacional e internacional, así como de la sociedad en su conjunto.
Además, se incluyen testimonios de mujeres supervivientes al cáncer mamario que a la vez son profesionales que buscan, desde sus experiencias, contribuir en la lucha contra esta enfermedad.

La incidencia de cáncer mamario y la creciente carga de muertes que ocasiona, es un problema mundial como se discute en el artículo elaborado por Porter. Hasta el momento, ningún país ha revertido el aumento en el número de casos, lo cual indica que los factores de riesgo que lo determinan no se han modificado. $\mathrm{Al}$ respecto, consistentemente se ha documentado que la edad temprana a la menarca, la nuliparidad o la edad tardía al primer embarazo, la falta de lactancia y la edad tardía de la menopausia son características reproductivas que incrementan el riesgo de desarrollarlo, no obstante $7 \mathrm{de}$ cada 10 pacientes diagnosticadas no cuentan con dichas características, tal como se presenta en al artículo elaborado por Torres Mejía y Ángeles Llerenas.

Es posible que los hábitos dietéticos tengan un papel determinante en la etiología de esta enfermedad. Si bien los alimentos son fuente de nutrimentos deseables, la frecuencia en su consumo y la forma de cocinarlos puede conllevar a ingerir un exceso de grasa saturada por ejemplo, o bien ser el vehículo de compuestos carcinogénicos como es el caso de las aminas arómaticas que se forman al asar carne. Torres-Sánchez y cols., elaboraron una revisión extensa acerca del papel de la dieta y el cáncer mamario en Latinoamérica, donde se evidencia que la misma juega un papel importante en el desarrollo de dicho tumor. Es de gran complejidad evaluar las relaciones que existen al combinar y cocinar alimentos, lo cual constituye un reto para el diseño de programas de prevención primaria que podrían promover no sólo el consumo de frutas y verduras, sino también la práctica permanente de actividad física como se presenta en el artículo elaborado por Romieu y Lajous.

No menos importante resulta la susceptibilidad genética individual en el riesgo de cáncer mamario. Con la 
reciente decodificación del genoma humano y tecnología de avanzada se espera un desarrollo sustantivo en el mediano plazo que permita identificar mujeres con alto riesgo genético de cáncer mamario como lo describen Hidalgo-Miranda y Jiménez-Sánchez, e implementar programas de tamizaje poblacional, para detectar mutaciones como las ya conocidas en los genes BRCA1 y BRCA2 que se analizan en el artículo presentado por Narod.

En contraste con el incremento en la incidencia de cáncer mamario a nivel mundial, las tendencias en la mortalidad presentan variaciones importantes. Algunos países han logrado disminuir el número de muertes. Desafortunadamente, ese no es el caso para los países de Latinoamérica y el Caribe. A este último respecto, pese a la falta de registros de cáncer de mayor calidad, LozanoAscensio y cols., presentan un diagnóstico que muestra una tendencia creciente en el número de muertes por dicha neoplasia maligna. Si bien este aumento podría estar explicado por la falta de acceso a la mamografia y al tratamiento, Marina-Franco y cols., plantean a través de modelos matemáticos, que las mujeres mexicanas nacidas entre 1940 y 1955 tienen los mayores aumentos en la mortalidad por cáncer mamario en comparación con las nacidas después de ese período. Dicho periodo se caracteriza entre otros, por la introducción al ambiente de contaminantes químicos utilizados para diferentes fines durante la Segunda Guerra Mundial. Posteriormente, Palacio-Mejía y cols., agregan información que muestra como la mortalidad por cáncer mamario se concentra principalmente en los estados del norte de la República mexicana.

El examen clínico y la mamografía, son las principales herramientas para el diagnóstico de cáncer mamario. Los factores relacionados con la demanda, accesibilidad y calidad de la detección del cáncer mamario son motivo de análisis en este suplemento. En cuanto al examen clínico, López-Carrillo y cols., destacan que las mujeres jóvenes con menor escolaridad y estrato socieconómico residentes en áreas urbanas, acuden al examen clínico significativamente en menos ocasiones que aquellas con mayor escolaridad. Más aún, de acuerdo a Knaul y cols., en 2006 sólo el 22\% de las mujeres de 40 a 69 años se sometió a una mamografía en el último año, lo cual es una proporción muy baja y mayor desde el año 2000. La situación es considerablemente peor entre mujeres de menor nivel socio-económico lo cual se ilustra con el dato sobre la población indígena donde tan sólo 1 de cada 10 reporta haber tenido una mamografía, como lo muestran Sosa-Rubí y cols.

Los factores y barreras que explican por qué un porcentaje tan reducido de la población llega a la detección temprana son de la oferta y de la demanda. Destaca la falta de sensibilización de las mujeres ante este problema. En particular, como lo muestran Nigenda y cols. y Unger-Saldaña e Infante-Castañeda, existen grandes deficiencias en la información sobre cáncer mamario no sólo entre la población general sino también entre los proveedores de servicios de salud.

Los países Latinoamericanos enfrentan un gran reto en extender la detección temprana y reducir la proporción de casos detectados en fases tardías cuando la probabilidad de supervivencia es mucho más baja y los tratamientos requeridos son más caros e invasivos. Se presenta la experiencia canadiense en detección temprana por Mai y cols., quienes enfatizan la necesidad de monitorear cercanamente los componentes específicos, en particular en el programa de tamizaje de cáncer mamario en ese país. Martínez y cols. analizan esta situación para el caso de México. La falta de programas exitosos de detección temprana y las inequidades existentes en el acceso al tratamiento de cáncer mamario, se ilustran para el caso de Colombia por parte de Velásquez y cols.

Entre más tardío es el diagnóstico más costoso el tratamiento respectivo, círculo vicioso que se demuestra para el caso del IMSS por Knaul y cols. y para el Instituto Nacional de Cancerología por Mohar y cols. Utilizando modelos econométricos, Valencia-Mendoza y cols., identifican en base a la edad de inicio de la mamografía, el porcentaje de cobertura y la periodicidad, las características idóneas de un programa de tamizaje que reduzca la detección de tumores tardíos y por ende los costos del tratamiento.

Son varios los artículos que destacan el papel de las organizaciones civiles organizadas a nivel nacional e internacional y de la sociedad en su conjunto. La preocupación de implementar programas de tamizaje y tratamientos exitosos para el cáncer mamario es un común denominador a nivel mundial de acuerdo a Gospodarowicz y cols. y requiere la colaboración no sólo internacional sino también multidisciplinaria y un enfoque en modificar las conductas de salud para prevenir el cáncer. Knaul y cols. destacan la importancia de considerar la detección del cáncer de mama en el marco de la iniciativas a nivel global sobre salud materna-infantil y reproductiva. Anderson y cols. a través del trabajo de la Iniciativa Global para la Salud de la Mama, resaltan la importancia de implementar programas integrales de prevención, detección y tratamiento acorde con las posibilidades y necesidades de cada país. En Durstine y cols. se resalta la importancia de aumentar el liderazgo a nivel de la sociedad civil para combatir esta enfermedad y para incentivar la participación de los pacientes en este movimiento. Existe una necesidad de que los grupos 
organizados trabajen conjuntamente con expertos en medicina y con líderes de acuerdo a Koon y cols. Este tipo de colaboración y acción por parte de la sociedad en su conjunto es necesario para promover cambios en el sistema de salud que resulten en una mejora de los servicios y en consecuencia de la calidad de vida como lo discuten Maza-Fernández y Vecchi-Martini.

El volumen dimensiona el reto que representa el cáncer de mama para la salud, los sistemas de salud y la sociedad. Quedan todavía muchos espacios de investigación escasamente explorados no solamente en la ciencia básica y el comportamiento de la enfermedad, sino también en las respuestas a desarrollar e implementar en el ámbito de los sistemas de salud y en cuanto al impacto en la sociedad incluyendo la pareja, la vida familiar y en el desarrollo profesional de la mujer diagnosticada con cáncer mamario.

Sin duda, los sistemas de salud enfrentarán un enorme desafío, que ha sido ampliamente descrito en este número especial de Salud Pública de México, que compromete a los tomadores de decisiones a ofrecer una respuesta inmediata con la práctica de medicina basada en evidencia. Su abordaje requiere de un enfoque integral que abarca los factores causales y de riesgo, la prevención, la detección y el tratamiento médico, además del impacto en las mujeres y los hombres, las familias y las parejas que viven con la enfermedad.

A lo largo del volumen aparece un mensaje común: la importancia de involucrar a todos, incluyendo los hombres, en el combate a esta enfermedad. Los sistemas sociales,la familia, los sectores de salud y educación y las economías, tienen que aplicarse a la tarea de atenuar el impacto que el cáncer de mama pudiera tener a corto y largo plazo. A la vez, se debe enfrentar esta gran tarea con la seguridad de que los avances que se pueden lograr en el combate al cáncer de mama tendrán beneficios en la lucha para alcanzar mejor salud, un trato más digno y servicios de salud de mayor calidad, sistemas de salud más eficaces y más equidad de género a nivel de la sociedad en su conjunto.

Finalmente, queremos agradecer profundamente las contribuciones de cada una de las personas que hicieron posible la realización de este suplemento que es una muestra del dolor, del interés, del trabajo e ideas que se están generando para combatir el cáncer mamario. En el ámbito tanto profesional como personal han sido muchos y muchas los amigos y colegas que han contribuído directa e indirectamente a lograr esta publicación, no quisiéramos omitir ningún nombre de esa extensa lista, y a todos les extendemos nuestro más sincero reconocimiento.

A nivel institucional, agradecemos el apoyo financiero del Instituto Carso de la Salud a través del programa 'Cáncer de mama: tómatelo a pecho'; la contribución del Observatorio de la Salud, proyecto conjunto del Instituto Carso de la Salud y de la Fundación Méxicana para la Salud a través de su Consejo Promotor Competitividad y Salud; la colaboración con la Seattle Cancer Care Alliance, el Fred Hutchinson Cancer Research Center, la Breast Health Global Initiative, la American Cancer Society, Cancer Care Ontario, el Partnership for Maternal, Newborn and Child Health (en OMS), el Instituto Nacional de Medicina Genómica, el Instituto Nacional de Perinatología, y el Instituto Nacional de Cancerología, y en especial, el patrocinio y el arduo y generoso trabajo de múltiples investigadores de la Fundación Mexicana para la Salud, el Instituto Nacional de Salud Pública y de los integrantes del grupo editorial de Salud Pública de México.

Felicia Marie Knaul, ${ }^{(1)}$ Lizbeth López Carrillo, Eduardo Lazcano Ponce, ${ }^{(2)}$ Héctor Gómez Dantés, ${ }^{(3)}$ Isabelle Romieu, ${ }^{(2)}$ Gabriela Torres. ${ }^{(2)}$

(1) Cáncer de mama: Tómatelo a Pecho y Observatorio de la Salud. Instituto Carso de la Salud y Fundación Mexicana para la Salud. México.

(2) Instituto Nacional de Salud Pública.

(3) Fundación Mexicana para la Salud, Observatorio de la Salud. México. 